\title{
Innovación en presas de relaves: almacenamiento con geotextiles Optimización de almacenamiento Presa de Relave Chocho
}

DOI: $10.46932 / \mathrm{sfjdv} 2 \mathrm{n} 2-210$

Received in: March 1st, 2021

Accepted in: May 30th, 2021

\author{
Boris García Fontana \\ Gran Colombia Gold-Maccaferri de Colombia, Colombia, Bogotá \\ E-mail: boris.garcia@grancolombiagold.com.co \\ Gerardo Fracassi \\ Gran Colombia Gold-Maccaferri de Colombia, Colombia, Bogotá \\ E-mail: g.fracassi@maccaferri.com
}

\section{RESUMEN}

La empresa Gran Colombia Gold Segovia, sucursal Colombia, actualmente, tiene una operación minera en el interior de los límites del título minero RPP 140, la cual está situada entre los municipios de Remedios y Segovia, Departamento de Antioquia - Colombia. Se realizó una propuesta para la optimización del proyecto, donde estas presas fueran construidas por el mismo relave producido en la mina. Así utilizando el material lodo que tenían en el sitio. Se realizó un estudio de viabilidad técnica superando los retos generales por temas hidráulicos, geotécnicos y ambientales.

Palabras Clave: Ingeniería, Innovación, Motor de un Cambio Sostenible, sostenibilidad ambiental, relaves.

\section{INTRODUCCIÓN}

La Minería en Colombia enfrenta año a año varios retos, entre estos mejorar su competitividad, lograr una mayor certeza de crecimiento en el país y fortalecer su reputación en las comunidades. Para mejorar su competitividad, lo que se busca es realizar un mayor aprovechamiento de los espacios limitados que tienen en las zonas mineras, logrando aumentar sus producciones, innovando día a día en métodos que involucren técnicas y soluciones de construcción que permitan la protección del medio ambiente.

El presente trabajo mostrará un caso, desde la problemática, proceso y solución empleando Geotubos, logrando la conformación de una presa llenados con el mismo material de relave de producción de la Mina.

\section{ANTECENDETES DEL PROYECTO:}

La empresa Gran Colombia Gold Segovia, sucursal Colombia, actualmente, tiene una operación minera en el interior de los límites del título minero RPP 140, la cual está situada entre los municipios de Remedios y Segovia, Departamento de Antioquia - Colombia. 
El principal aporte de mineral de oro proviene de las minas subterráneas El Silencio, Providencia y Sandra K y un segundo aporte de la operación de las unidades de pequeña minería dentro de la propiedad de GCG.

El mineral, se procesan en la Planta María Dama y Los relaves producto del beneficio son bombeados hasta el depósito EL Chocho.

El proyecto original contempla dos embalses generados por 2 presas de tierra, denominadas: Almacenamiento de relaves superior e inferior cómo se muestra en la

Ilustración 1. Ambos embalses, contemplaban una capacidad de almacenamiento de 1,5 millones m3. Sin embargo, debido a las características de gobernabilidad del terreno y a las condiciones económicas de la inversión, se optimizó el área dentro del Almacenamiento superior y el proceso de disposición fue denominado: Contención de relaves.

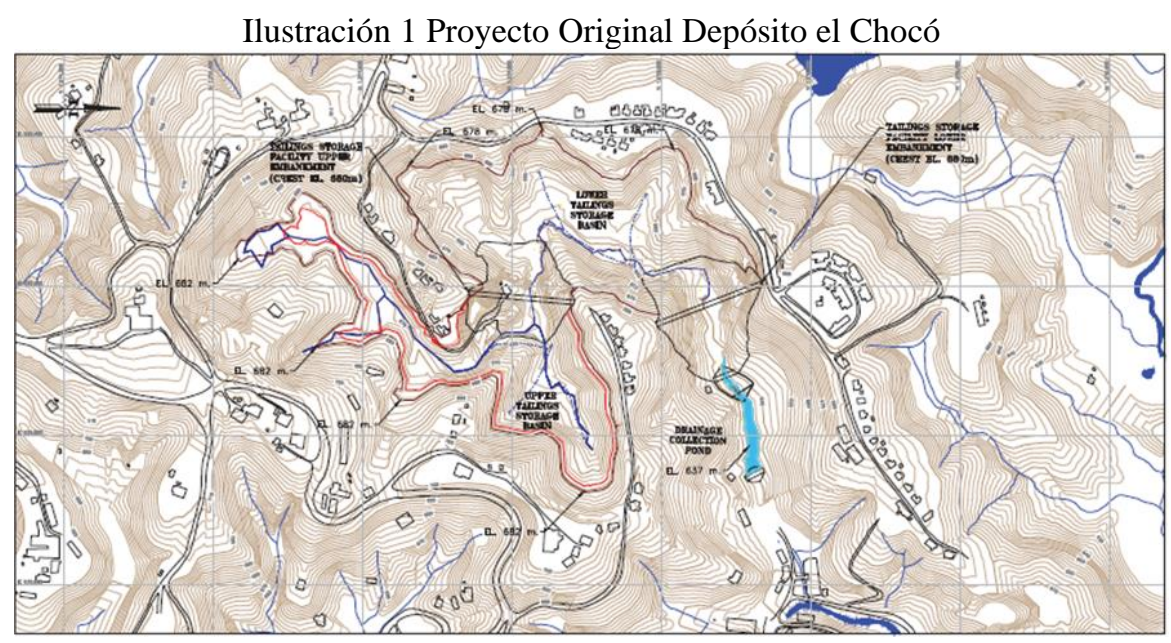

En el Almacenamiento de relaves superior se definieron las fases 1A, 1B y 1C, esquematizadas en la Ilustración 2. Actualmente, la fase 1B está $100 \%$ de su capacidad total y las fases $1 \mathrm{~A}$ y $1 \mathrm{C}$, en construcción. Para tecnificar el proceso de disposición de lodos (uso de tubos Geotextiles y filtro Prensa) en la fase 1B se utilizaron 33 Geotubos confeccionados con geotextiles, en la fase 1A se utilizaron 61 geotubos, material que facilita el drenaje del agua y retiene el relave seco para finalmente crear un dique de contención con los mismos. 


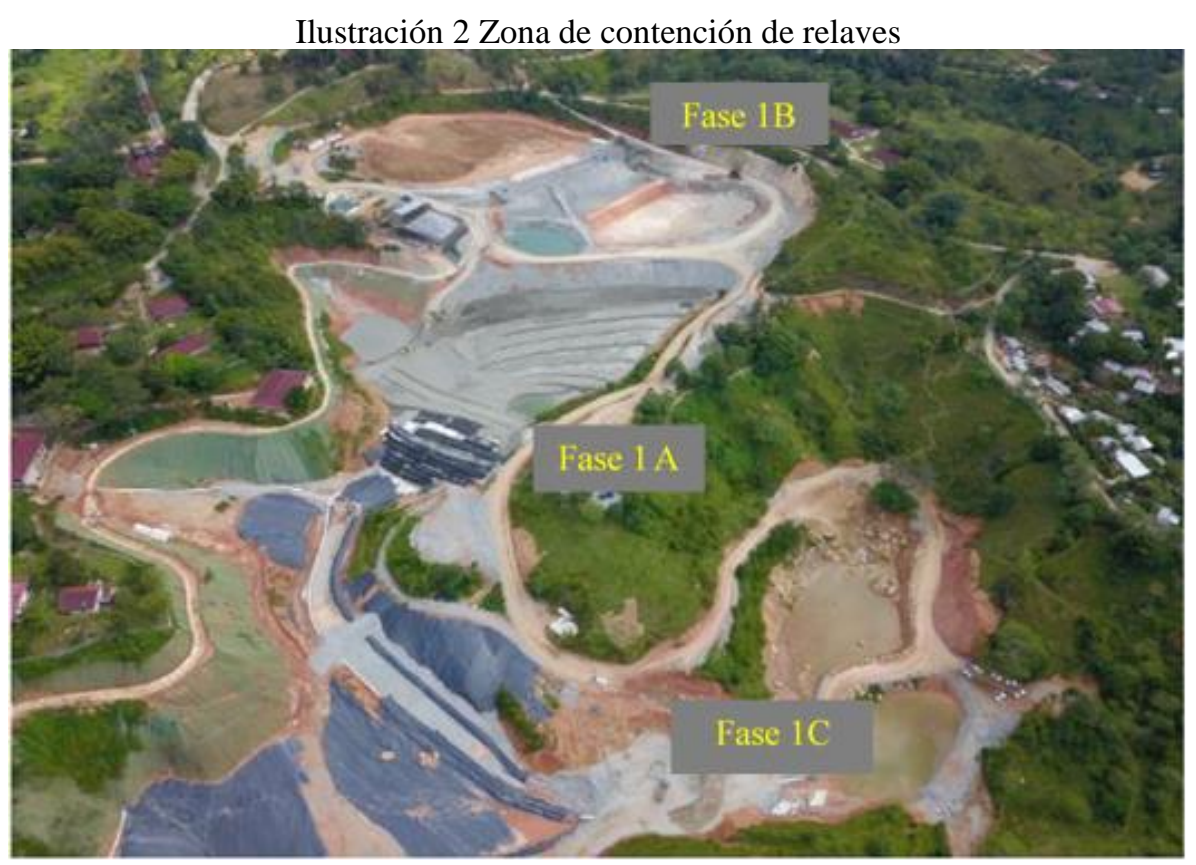

\section{DESCRIPCIÓN Y ANÁLISIS DE LA ESTRUCTURA}

Para realizar el análisis del proyecto se revisan dos opciones de las cuales la opción con mejor aprovechamiento del material de relave que la mina produce es la conformada en Geotubos por medio de deshidratación de lodos.

El Mactube es un tubo fabricado por la empresa Maccaferri con geotextil tejido de alta tenacidad, inerte a la degradación biológica y resistente a los ataques químicos. El funcionamiento del Mactube es al recibir el lodo en su interior, éste permite el flujo del líquido a través de los poros del geotextil tejido, el cual retendrá las partículas sólidas, reduciendo el tenor de humedad y consecuentemente generando un líquido clarificado. después del completo llenado y finalizado el proceso de deshidratación y consolidado del lodo seco en su interior puede ser dispuesto de la manera proyectada.

Para realizar el análisis del uso de Geotubos en el proyecto se tuvieron unas etapas principales:

\subsection{ANÁLISIS DE RELAVE EN LABORATORIO}

Se realizó análisis en laboratorio del relave que tenía Gran Colombia Gold para la certificación del funcionamiento de la tecnología, la definición de los polímeros a utilizar y las particulares químicas, físicas e hidráulicas del material a ser deshidratado. El polímero utilizado en la construcción de las presas es el Aniónico, resultados que se muestran en la Ilustración 3. 
Ilustración 3 Ensayo Test De Jarras

\begin{tabular}{|c|c|c|}
\hline \multicolumn{3}{|c|}{$\begin{array}{l}\text { PRUEBA DE JARRAS } \\
\text { (introducirvalores en celdas amarillas) }\end{array}$} \\
\hline \multicolumn{3}{|c|}{ Proyecto: Gran Colombia Gold } \\
\hline \multicolumn{3}{|l|}{ FECHA: $26 /$ Sep/2017 } \\
\hline \multicolumn{3}{|l|}{ REALUZADA EN: SEGOVIA ANTOQUIA } \\
\hline \multicolumn{3}{|c|}{ PRUEBA \#2 - GDT (Polimero Anionico) } \\
\hline ITEM & CANTIDAD & UNIDAD \\
\hline \multicolumn{3}{|l|}{ 1.Volumen lodo } \\
\hline $\mathrm{ml}$ de lodo & 20000 & $\mathrm{ml}$ \\
\hline ml de agua adicionada & 0 & $\mathrm{ml}$ \\
\hline \multicolumn{3}{|l|}{ 2.Volumen polímero } \\
\hline mil de agua & 1000 & $\mathrm{ml}$ \\
\hline m3 de agua & 0.001 & $\mathrm{~m}^{3}$ \\
\hline gr de polimero & 5 & gr \\
\hline Tipo de polimero & Anionico & N/A \\
\hline Nombre comercial polimero & Proestol 2641 & N/A \\
\hline Concentración en polimero & 5000 & PPM \\
\hline \multicolumn{3}{|l|}{ 3.Volumen dosifieado } \\
\hline $\mathrm{ml}$ de polimero inyectado & 100 & $\mathrm{ml}$ \\
\hline $\mathrm{m} 3$ de polimero inyectado & 0.0001 & $\mathrm{~m}^{3}$ \\
\hline Concentración en polimero inyectado & 25 & PPM \\
\hline \multicolumn{3}{|l|}{ 5.Volumen de clarificado } \\
\hline ml de clarificado a punto de goteo & 16000 & $\mathrm{ml}$ \\
\hline \% de Humedad Removida & $80 \%$ & $\%$ \\
\hline Tlempo de clarificado & 12 & Min \\
\hline
\end{tabular}

\subsection{DIMENSIONAMIENTO DEL PROYECTO}

Con los resultados de laboratorio se dimensiono el proyecto y se proyectó una presa con Geotubos que fueran apropiados para el lodo que se tenía en el proyecto. Adicional se verifica la estabilidad de la presa geotécnicamente.

Para la verificación de la estabilidad de la presa, se requiere saber el tipo de suelo que se tiene en la fundación, el relleno estructural de la presa en este caso el peso específico de relave. En la

Tabla 1, se muestran las propiedades de los materiales con los que fueron analizados la estabilidad de la presa.

Tabla 1 Propiedades de materiales para Análisis de Estabilidad

\begin{tabular}{|l|l|l|l|}
\hline Material & Cohesión(kN/m2) & Angulo de Fricción $\left(^{\circ}\right)$ & $\begin{array}{l}\text { Peso } \\
(\mathrm{kN} / \mathrm{m} 3)\end{array}$ \\
\hline Fundación-Macizo Rocoso & 150 & 50 & 20 \\
\hline Relleno del Geotubo & 45 & 0 & 18 \\
\hline Tailings & 0 & 10 & 18 \\
\hline
\end{tabular}

\subsection{ANÁLISIS DE ESTABILIDAD:}

Con los datos anteriores, se realizó el dimensionamiento de la presa y el análisis de estabilidad global de la presa. El dimensionamiento propuesto por la empresa Maccaferri se esquematiza en la Erro! Fonte de referência não encontrada. tanto en Sección Esquemática cómo en planta por cada Nivel de altura. 
Ilustración 4 Diagrama de la presa de Relave "The Chocó"

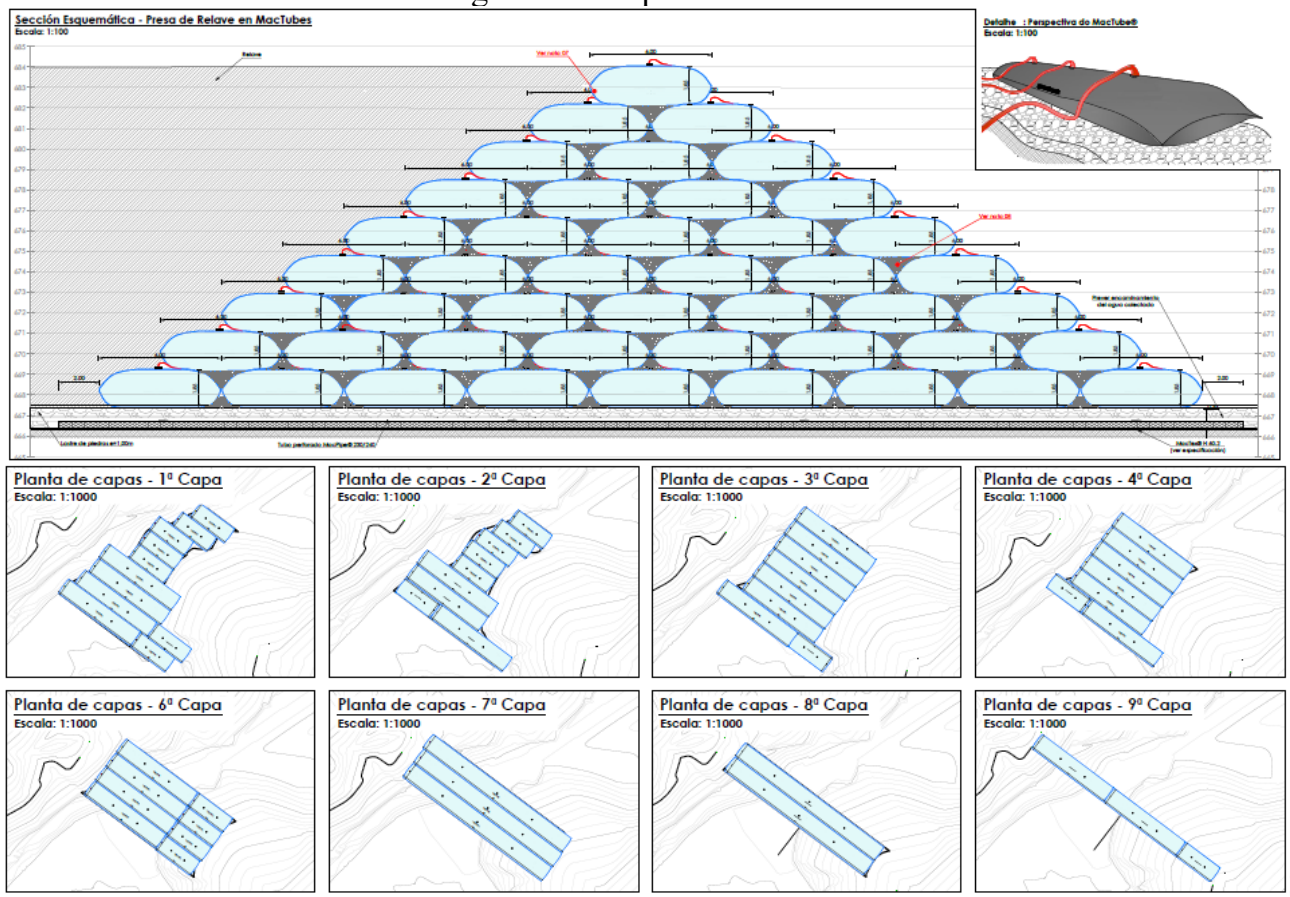

Seguidos de esto se realizó el análisis de estabilidad de la presa con un resultado final de 1.250 para una condición pseudoestática.

Ilustración 4 Estabilidad Global Presa de Relaves-Corrida MacStars

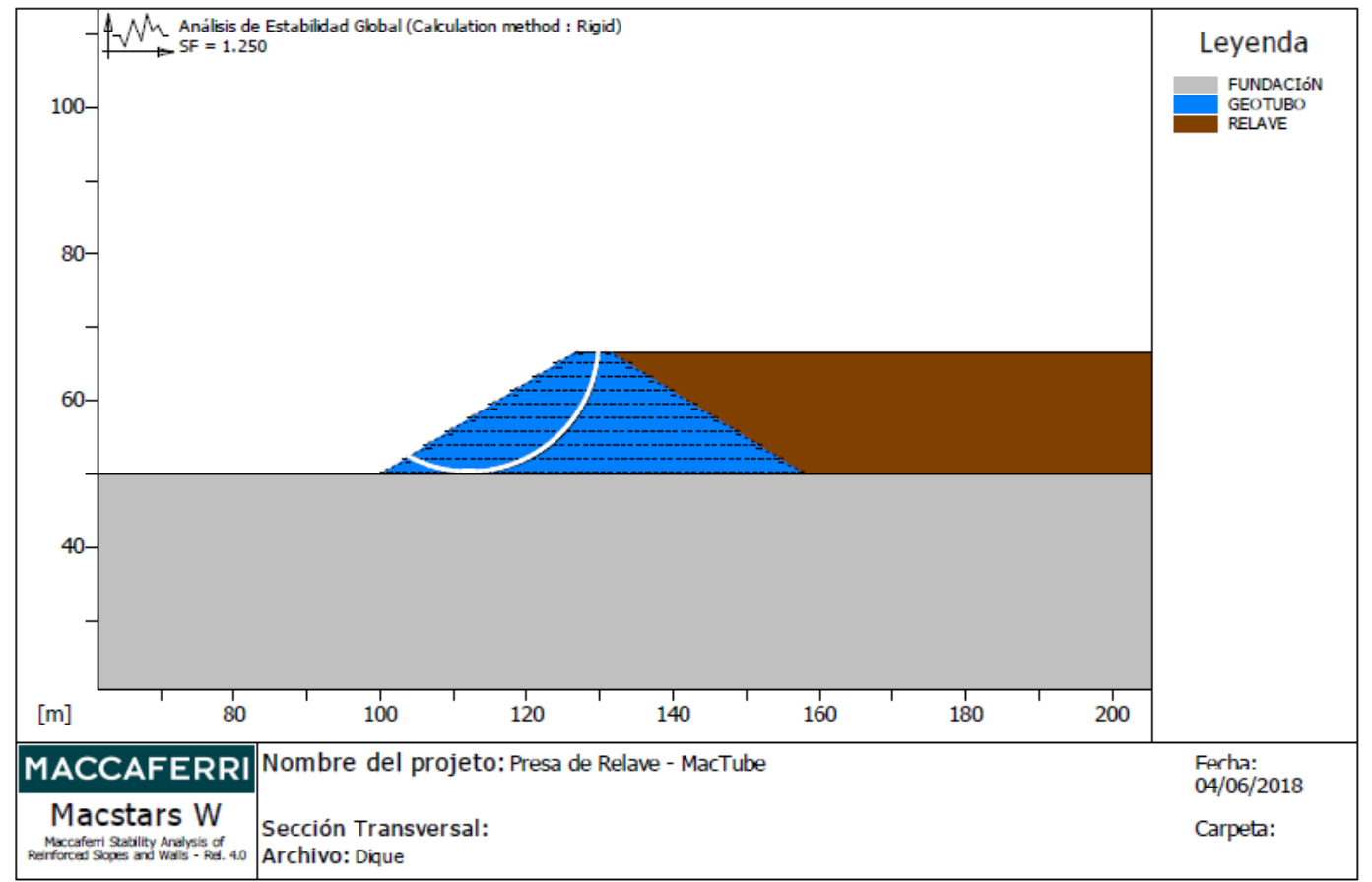




\subsection{CAPACIDAD DE ALMACENAMIENTO OPTIMIZADA}

Con estos análisis y basados en la topografía de la zona y el uso del software AutoCad Civil 3D se definieron los planos con curvas de nivel a cada metro, los cuales, indican la capacidad volumétrica a medida que se va llenando el depósito de relaves. En la Tabla 2 se indican las capacidades de almacenamiento optimizada del proyecto El Chocho para sus diferentes fases.

Tabla 2 Capacidad de Almacenamiento

\begin{tabular}{|c|c|c|c|c|c|}
\hline ETAPAS DEL PROYECTO & $\begin{array}{c}\text { Inicio de } \\
\text { deposición }\end{array}$ & $\begin{array}{c}\text { Fin de } \\
\text { deposición }\end{array}$ & $\begin{array}{c}\text { Duración } \\
\text { (días) }\end{array}$ & $\begin{array}{c}\text { Capacidad } \\
\text { (m3) }\end{array}$ & $\begin{array}{c}\text { Volúmen } \\
\text { Almacenamiento } \\
\text { (m3) }\end{array}$ \\
\hline Contención Fase 1B (Bombeo) & $2018 / 04 / 13$ & $2019 / 05 / 01$ & 383 & 240,000 & 240,000 \\
\hline Contención Fase 1A (Geotubos) & $2019 / 05 / 01$ & $2019 / 11 / 10$ & 194 & 140,000 & 140,000 \\
\hline Contención Fase 1C (Filtroprensado) & $2019 / 11 / 10$ & $2022 / 07 / 09$ & 972 & 810,000 & 810,000 \\
\hline \multicolumn{7}{|c|}{} & $2025 / 08 / 07$ & 1124 & $1,300,000$ & $1,300,000$ \\
\hline Almacenamiento inferior (Filtroprensado) & $2022 / 07 / 09$ & \multicolumn{7}{|c|}{ Total Fase 1 Fase 2 } & $\mathbf{1 , 3 0 0 , 0 0 0}$ \\
\hline
\end{tabular}

La Fase 1 del Proyecto de relaves El Chocho, proyectada para una capacidad inicial de $497.00 \mathrm{~m} 3$ alcanzará una capacidad de $1.190 .000 \mathrm{~m} 3$ con la tecnificación de su proceso constructivo (relaves filtro prensados y geo-tubos). La optimización representa un aumento de más del 100\% de capacidad del proyecto original.

Después de analizada la estructura y ser validada por los expertos especialistas, se inició la construcción. Se realizaron obras adicionales y las zonas que hacen parte de la presa para la conformación de la misma:

\section{OBRAS EN ZONAS DE ALMACENAMIENTO}

\subsection{DIQUE TOMA Y DRENAJE DE AGUAS SUBSUPERFICIALES}

La quebrada Peñitas tiene su punto de nacimiento en el área de contención Fase 1B, la misma, es captada por un dique toma mostrado en la Ilustración 5 y a través de una tubería principal instalada sobre una cama drenante en el lecho de la quebrada de la

Ilustración 6a es entregada al final del área del proyecto en el cauce natural de la quebrada, en esta misma tubería confluyen un sistema de drenes en forma de espina de pescado que captan las aguas subsuperficiales del embalse mostrados en la

Ilustración 6 b. 
Ilustración 5 Dique Toma, durante la construcción

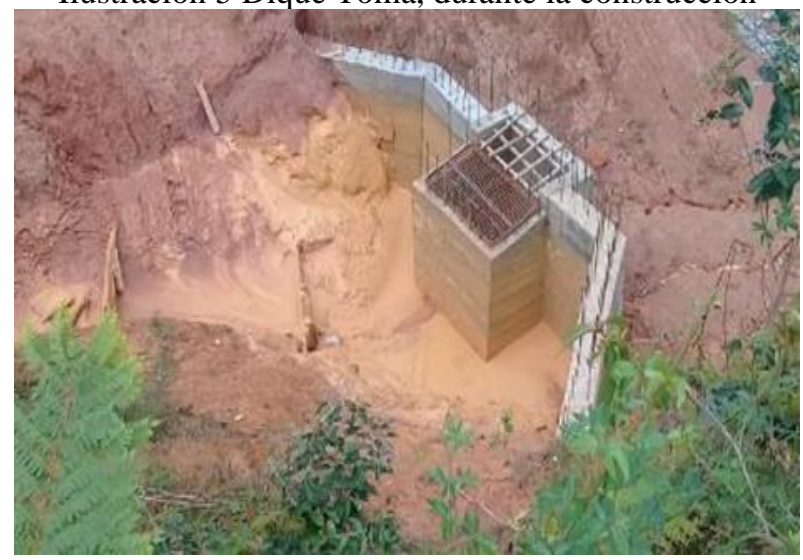

Ilustración 6a y 7b. Drenaje de aguas subsuperficiales
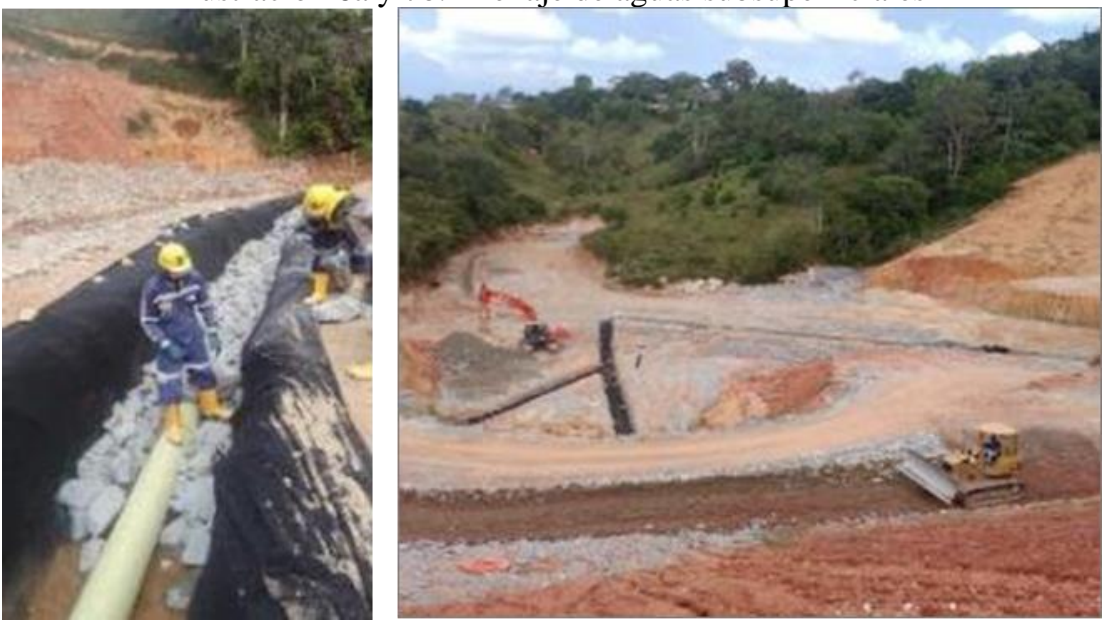

\subsection{PROTECCIÓN DEL SUBSUELO}

El subsuelo de las áreas de contención de relaves es protegido de la posible infiltración de aguas con la colocación de una capa de arcilla de 50 centímetros compactada como se muestra en la Ilustración 7. El sistema de drenaje de las aguas subsuperficiales queda protegido bajo la capa impermeable.

\subsection{MANEJO DE AGUAS DE LOS RELAVES}

El agua producto de los relaves se capta a través de un sistema de subdrenaje en forma de espina de pescado ubicado sobre la capa impermeable mostrado en la Ilustración 7, el cual, recoge las aguas y las entrega a una estructura de decantación que ayuda a su evacuación para ser entregadas posteriormente, en la planta de beneficio María Dama, y así, ser reutilizada en el proceso.

Por razones constructivas, este sistema de drenaje no fue ejecutado en la fase $1 \mathrm{~B}$, donde se sustituyó por una piscina que recibe las aguas filtradas por un rock Dam, esta zona de almacenamiento y las entrega directamente a la planta de beneficio. Ver ilustración 6. 


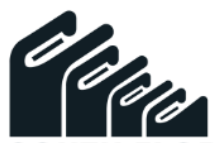

Ilustración 6 Fases del Proyecto el Choco

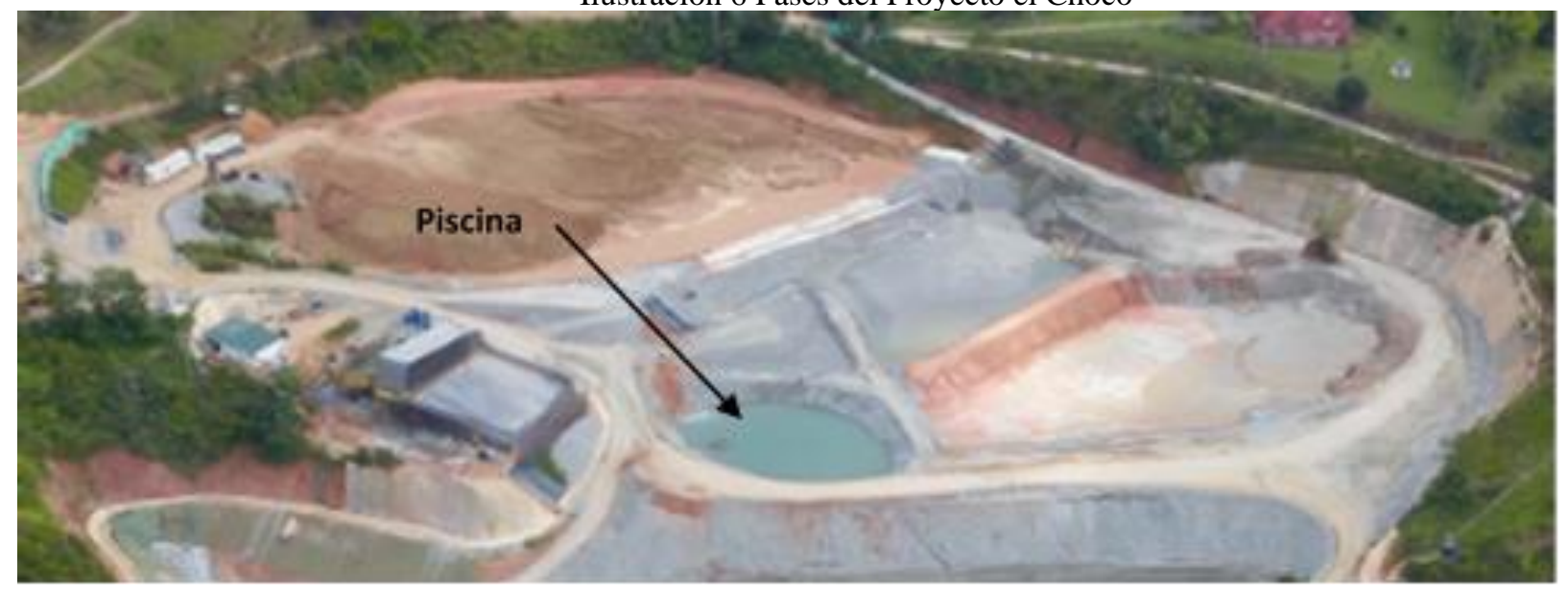

Ilustración 7 Manejo de Aguas para reproceso Fase 1A

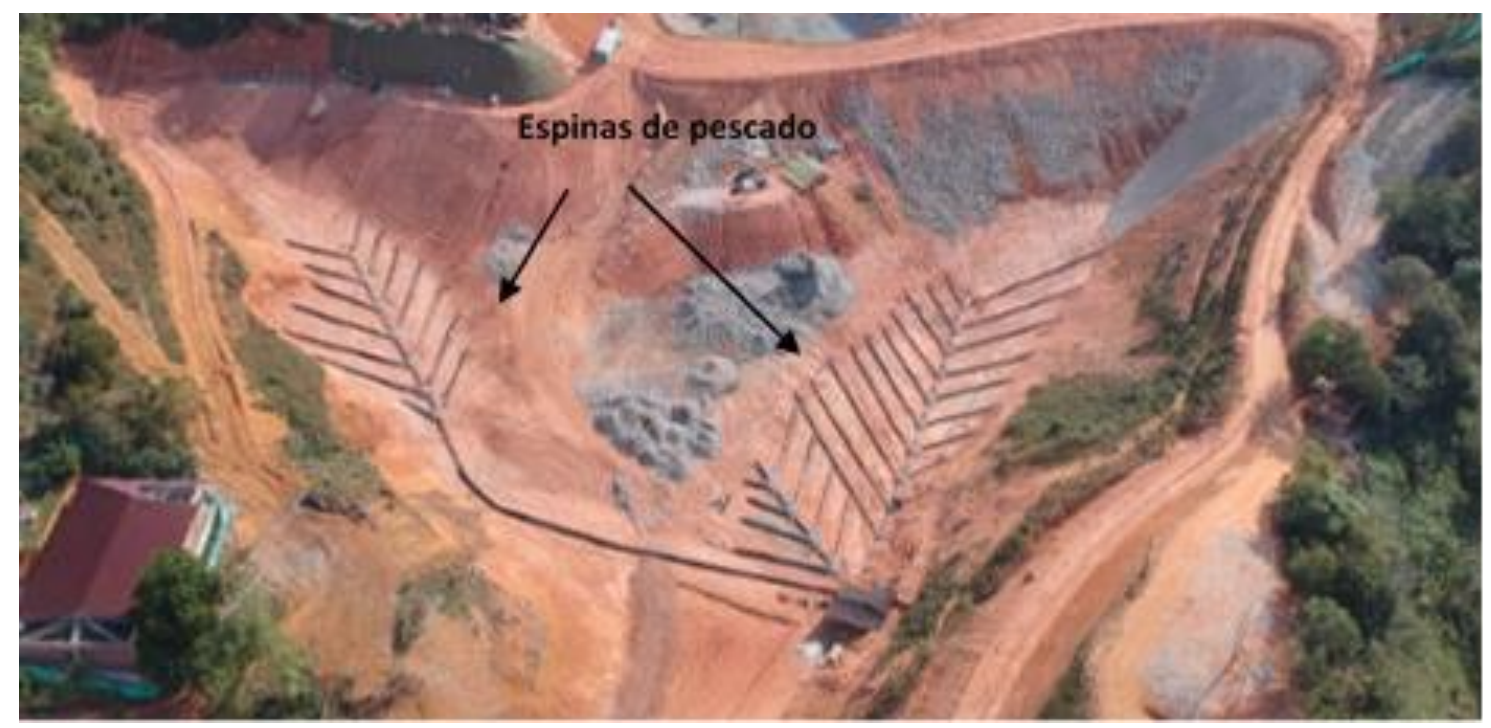

\subsection{AGUAS DE CONTACTO}

Las aguas de contacto de precipitación con el relave serán captadas por unos canales perimetrales con dirección a la piscina de colección. El muestreo indica que esta agua es apta para el vertimiento. Por lo tanto, pasará por rebose hacia las escorrentías naturales de la quebrada peñitas. 
Ilustración 8 Fases del proyecto el Chocó

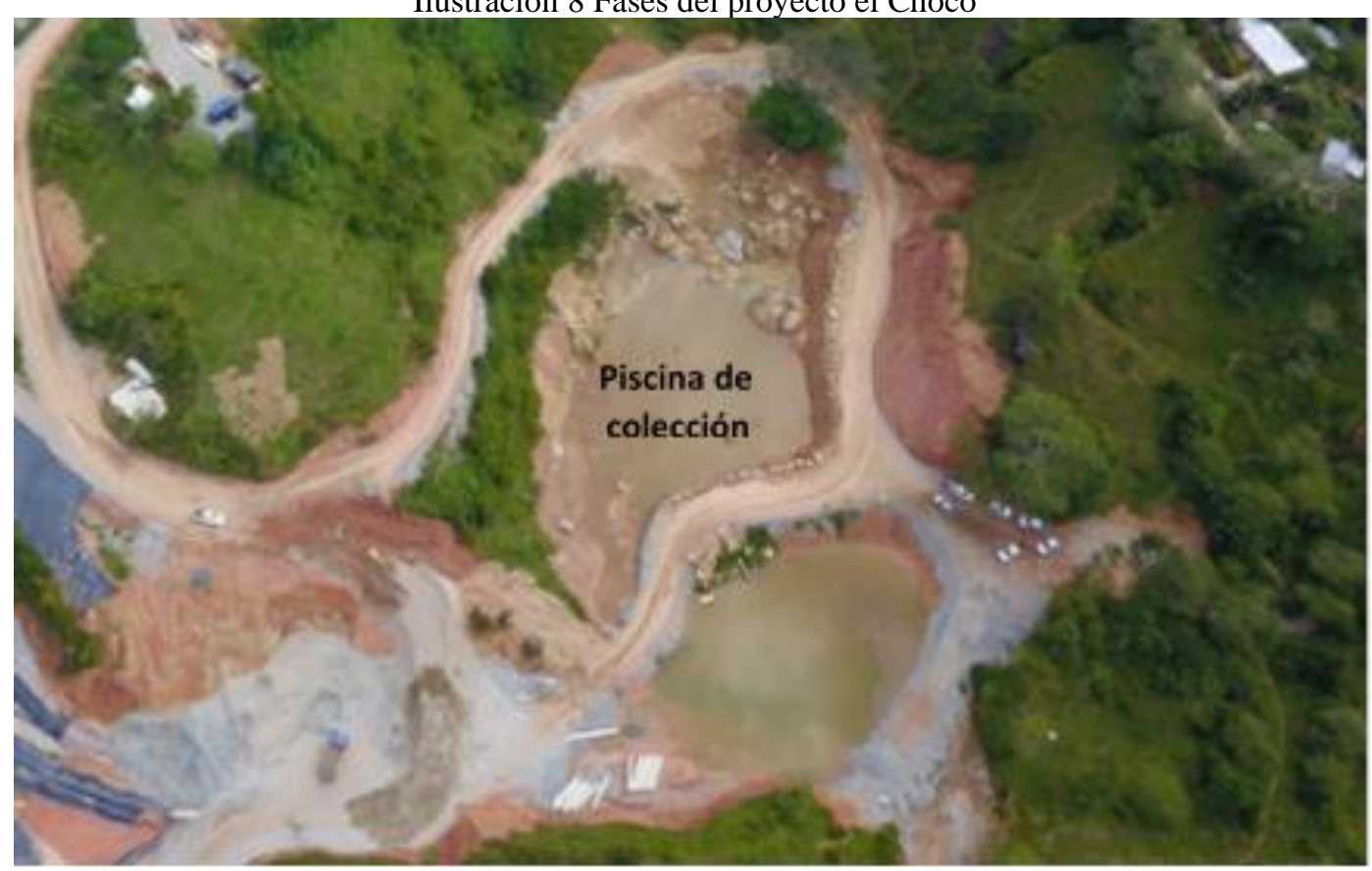

\subsection{REQUERIMIENTOS AMBIENTALES}

Por último y lo más importante la empresa Gran Colombia Gold Segovia en cumplimiento de los compromisos ambientales ha ejecutado, compensación de árboles por áreas deforestadas, canalización de las aguas de lluvia que escurren en las zonas de almacenamiento del proyecto (no contacto) y el seguimiento a la calidad del agua de la quebrada Peñitas.

\section{PROYECTO FINALIZADO}

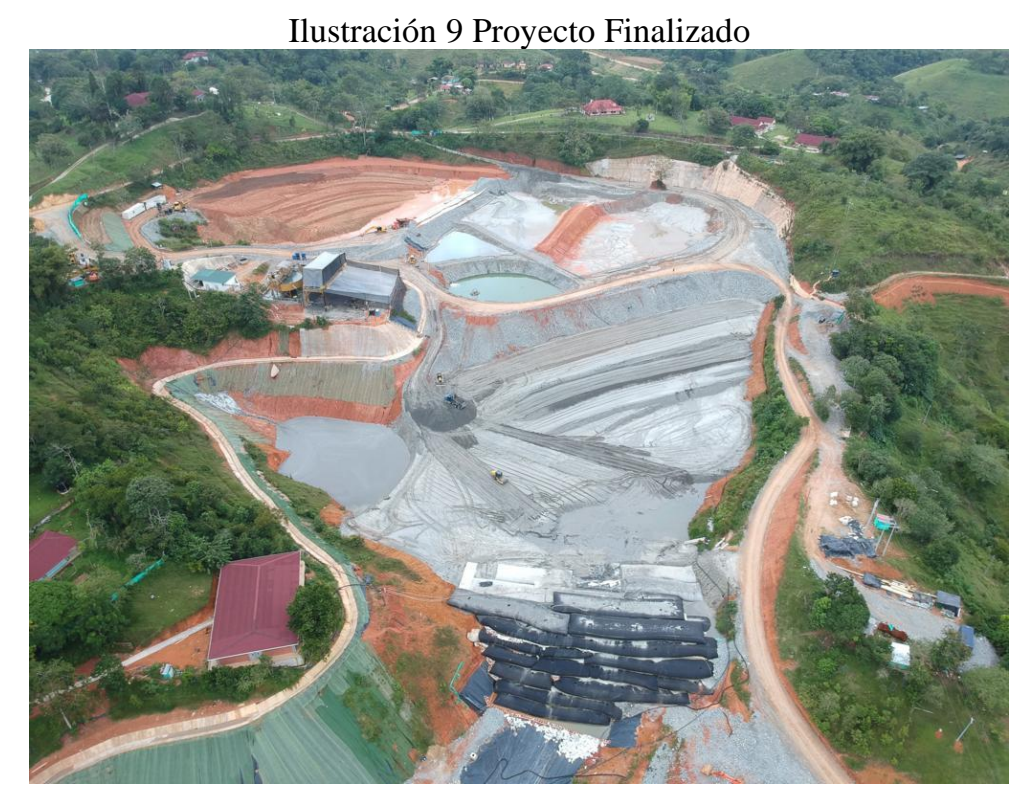




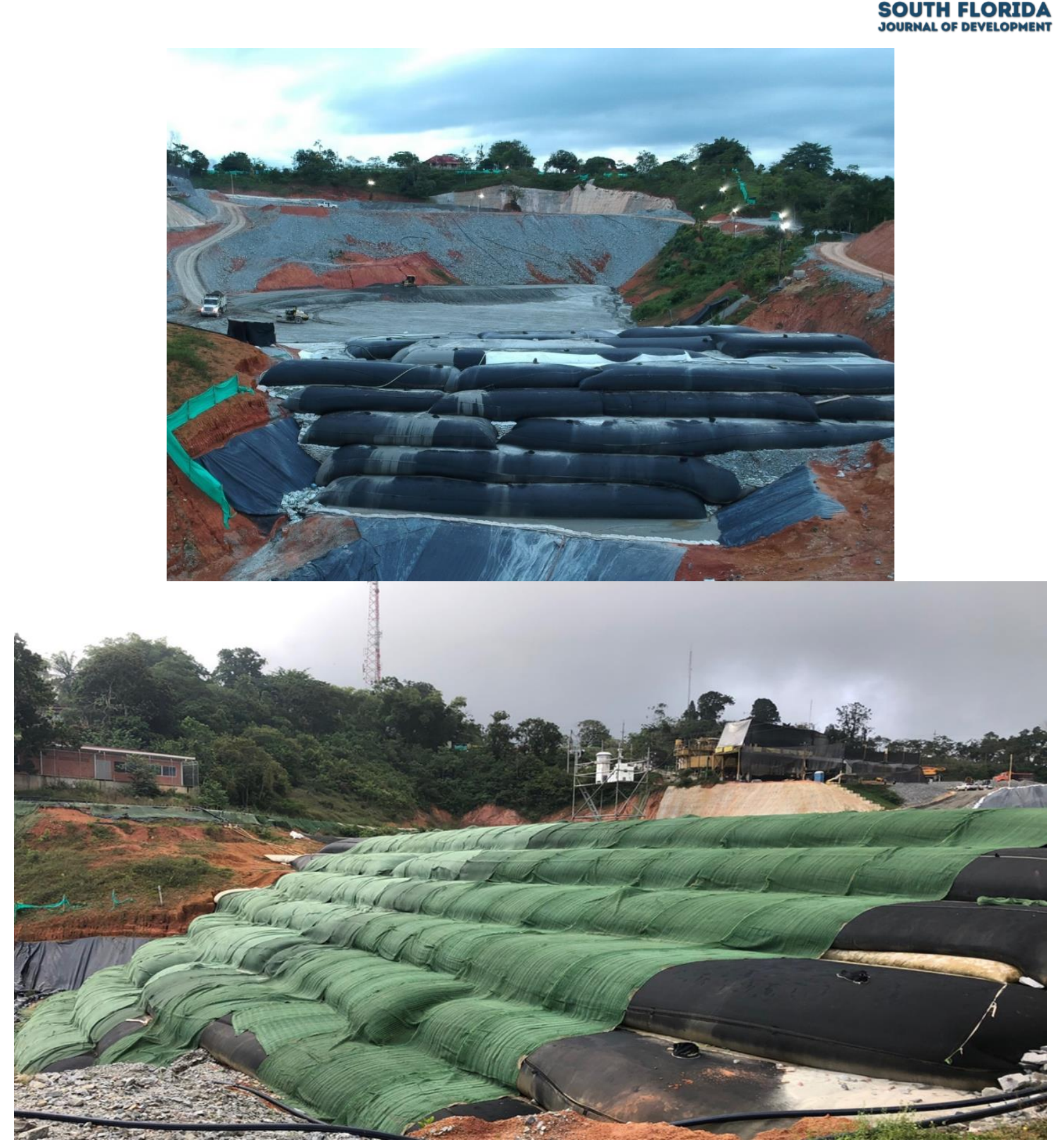




\section{REFERENCIAS}

MACSTARS W-Maccaferri 1998-Release 4.0 (Software de analysis para contentions) 